\title{
Obtención de un híbrido de frijol arbustivo para una cosecha mecanizada
}

Carlos Muñoz Ruiz'

Fecha de recepción: 7 de setiembre del 2011 Fecha de aceptación: 12 de diciembre del 201 I

Muñoz, C. Obtención de un híbrido de frijol arbustivo para una cosecha mecanizada. Tecnología en Marcha. Vol. 25, No 2. Abril-Junio 2012. Pág 2l-3l.

I. Ingeniero agrónomo, doctor en Sistemas de Producción Tropical Sostenible. Catedrático y profesor investigador, Escuela de Agronomía, Tecnológico de Costa Rica, sede regional San Carlos. Correo electrónico: cmunoz5 I @gmail.com 


\section{Resumen}

En el estudio se evaluaron tres variedades de frijol negro (Brunca, Guaymí y Talamanca), obtenidas en la estación experimental Fabio Baudrit de la Universidad de Costa Rica, y una variedad (Negro Chapingo), obtenida en la Universidad Autónoma de Chapingo, México.

Estas variedades no presentaron las características genéticas deseadas para ser utilizadas como donadores y aceptores de polen para obtener un híbrido arbustivo y de floración determinada para su cosecha en forma mecánica en la zona norte del país.

Sin embargo, se lograron obtener cinco hibridos $\mathrm{FI}$ entre los cruces realizados, ellos son: BCh, ChT, TCh, BT y TB. Todos ellos presentaron en la F2 características genéticas de porte semiarbustivo (TII y TIII) y de floración indeterminada, no compatibles para la obtención de un híbrido adecuado para la cosecha de frijol mecanizada.

En este proyecto de investigación se utilizó el método de polinización dirigida o artificial y el cruce simple recíproco entre las variedades participantes y se hicieron 34 I polinizaciones entre sí, respectivamente.

\section{Palabras clave}

Frijol (Phaseolus vulgaris L), cosecha mecánica, cruces simples recíprocos, híbrido arbustivo, mejoramiento genético.

\begin{abstract}
Three black bean varieties (Brunca, Guaymi, Talamanca) obtained from the University of Costa Rica's Fabio Baudrit Experimental Station, and a variety (Negro Chapingo) obtained from the Autonomous University of Chapingo, Mexico, were evaluated.These varieties did not present the genetic characteristics desired for their use as donators and acceptors of pollen for obtaining a determinateflowering bush-type hybrid for use in mechanical harvesting in the Northen Zone of the country.
\end{abstract}

However, five FI hybrids were obtained in the crosses realized; these are: BCh, ChT,TCh, BT and TB. All of these presented, in F2, semi-bushing genetic characteristics with non-determinate flowering, which are non-compatible for obtaining a hybrid adequate for mechanized bean harvest. In this research project, directed or artificial pollination was used, and the simple reciprocal cross between the participant varieties, $34 \mathrm{Icross}-$ pollinations was performed.

Key words

Bean (Phaseolus vulgaris L), mechanical harvesting, simple reciprocal crosses, bush-type hybrid, plant breeding. 


\section{Introducción}

El frijol (Phaseolus vulgaris L) es uno de los granos más utilizados para consumo humano y forma parte de la dieta de los habitantes del continente americano desde tiempos anteriores y posteriores a la conquista, especialmente en la zona de Mesoamérica.

Este cultivo ocupa, junto con el arroz, un lugar importante en la alimentación y en la adquisición de proteína asequible a precios bajos y está presente en cualquier mesa sin distinción de clase social o etnia (Rojas, 1998).

En Costa Rica, este cultivo se encuentra mayormente en manos de pequeños productores, y en muy pocos casos de grandes y medianos productores, y se distribuye especialmente en la faja frijolera del norte del país, en las zonas de Los Chiles, Upala, San Carlos y Guatuso.

El área de siembra total de frijol en el país llegó a 16.769 hectáreas (ha) en el periodo 2008-2009, con un rendimiento de 0,62 toneladas métricas, ligeramente superior al del periodo 2007-2008, con 0,60 toneladas métricas. El Consejo Nacional de Producción (CNP) dispone en estos momentos de semilla certificada para sembrar unas 12500 ha de frijol negro (CNP, 2009).

El país consume especialmente frijol negro, con unas 4000 toneladas métricas mensuales, lo cual corresponde a 48000 toneladas métricas anuales, o sea un $65-70 \%$ de todo el frijol. El país cosechó en el periodo 2008-2009 apenas 970 I toneladas métricas de frijol negro, es decir, un 20\% (CNP, 2009).

Costa Rica se abastece de frijoles principalmente por importación, actividad que muestra un aumento creciente. En 1998 se importaron 24, I toneladas de frijoles negros con un valor de US\$19,9 millones. En 2002 la importación fue de 30,8 toneladas, con un costo de US\$17,6 millones. El grano negro ocupa el 83\% de la importación (Salazar, 2004).

El cultivo de frijol para el consumo interno es un rubro de primera necesidad para la dieta costarricense. En este momento, la actividad está sufriendo además por la crisis de los precios, problemas relacionados con pérdidas en la cosecha por condiciones climatológicas y la falta de mano de obra suficiente para realizar la recolección, la cual es totalmente manual.

Durante el periodo de cosecha, el exceso de humedad permite la germinación del grano dentro de la vaina y dificulta las labores propias de la recolección y el secado. Estas condiciones ocasionan pérdidas de postcosecha y son difíciles de controlar debido al clima propio de la zona Huetar Norte.

Es indispensable contar con un ambiente seco en el transcurso de la maduración o secamiento de la vaina, así como en el momento de la cosecha (Jiménez, 1990).

La cosecha requiere gran cantidad de mano de obra y en la actividad participan aproximadamente 45005000 familias, entre pequeños y medianos productores, siendo esta una excelente opción de empleo para la mano de obra familiar (Ledesma, 2000).

Este cultivo ocupa el $11 \%$ de la población económicamente activa (PEA) regional, estimada en 227205 habitantes, con un 52\% de mujeres y un 48\% de hombres, con áreas más o menos de 20 ha. El total de productores en la zona Huetar Norte en el periodo agrícola 99/00 fue de 3450 (Ledesma, 2000).

La relación área/mano de obra disponible es de 8,83 ha por productor, la cual es demasiado alta para poder hacerle frente con éxito durante los periodos pico de cosecha del grano, lo cual obliga a contratar mano de obra adicional, especialmente la nicaragüense, la cual es inconstante, efímera y disminuye las ganancias del productor.

Las pérdidas ocasionadas por la lluvia en post cosecha, en primer lugar, son incuantificables y por la escasez de mano de obra, en segundo lugar, obligan a tomar medidas para disminuirlas y favorecer al productor y, a la vez, a que el Estado no tenga que subvencionarlos con el Presupuesto Nacional en el Seguro de Cosecha hasta por el 75\% (CNP, 2009).

La necesidad de darle una solución a la situación de la producción de frijol en la zona norte del país valida el objetivo principal de esta investigación sobre el mejoramiento genético del frijol, con lo cual se solucionaría en parte el problema de falta de mano de obra y de los cambiantes estados climatológicos de la zona de influencia, al ofrecer una variedad de frijol apta para la cosecha mecánica.

\section{Objetivo general}

Obtener,mediante la hibridación dirigida,una variedad (hibrido) de frijol negro arbustivo de alta producción que pueda ser cosechada mecánicamente en la región Huetar Norte de Costa Rica. 


\section{Objetivos específicos}

I. Introducir en el país variedades de porte arbustivo que reúnan las condiciones morfológicas para ser cosechadas mecánicamente.

2. La hibridación dirigida $\bigcirc$ artificial mediante cruces simples, usando como madre la variedad introducida seleccionada más adecuada y como padre la o las variedades "criollas" más usadas por el productor de frijol en la zona y a la inversa para fijar los caracteres deseados (cruces recíprocos).

3. La selección individual y la evaluación del material híbrido segregante (F2), durante un mínimo de una generación, F3 ( I,0- I,5 años).

\section{Revisión bibliográfica}

El frijol es originario de Mesoamérica y América del Sur. Son plantas anuales, con un sistema radical bien desarrollado y de crecimiento muy rápido, compuesto de una raíz principal con muchas y muy ramificadas raíces secundarias situadas en la parte superior, cercana a la superficie; tallos delgados y débiles, angulosos, de sección cuadrangular a veces rayados de púrpura y de altura muy variable; el porte de las plantas está determinado por la forma y posición de los tallos: si el principal tiene una inflorescencia terminal, el desarrollo de los tallos se detiene pronto y la planta será "enana"; si el tallo no produce esta inflorescencia terminal aparecen otras axilares, la planta será "de enrame" (porte trepador o voluble) (Mateo, 196I).

Se han definido de acuerdo con este tipo de crecimiento los siguientes: tipo I, cuando es de tipo arbustivo; tipo II, cuando es semiarbustivo; y tipo III y tipo IV cuando tienden a ser postradas y con líneas guía o de enrame cortas y largas. Con respecto a la floración, se destacan dos tipos bien definidos: de floración determinada, cuando se inicia la aparición de las inflorescencias de arriba hacia abajo, una característica fundamental para la cosecha mecanizada; y de floración indeterminada, cuando la aparición de las inflorescencias comienza de abajo hacia arriba, característica no deseada para la mecanización (Monge, 1989).

El frijol es una planta autógama, es decir, de autopolinización, en la cual, por sus características de alta homocigocidad, todas las líneas que se pueden obtener mediante la polinización dirigida - controlada (hibridación) son líneas puras homocigotos, donde la segregación varietal está limitada a pocas generaciones y la aclimatación del cultivar es casi nula (Poehlman, 1987).

El carácter del crecimiento indeterminado es controlado por un gen dominante llamado Fin; las plantas son indeterminadas cuando son homocigotos (Fin Fin). El hábito de crecimiento determinado es dominante solo cuando son homocigotos por el alelo recesivo (fin fin) (López et al., 1985).

Cuando una planta de frijol indeterminada se cruza con una planta determinada, el híbrido $\left(F_{1}\right)$ es indeterminado; cuando 100 semillas de este híbrido $\left(F_{2}\right)$ se siembran, aproximadamente 75 de las plantas serán indeterminadas y 25 determinadas (3:I) (López et al., 1985).

El ciclo vegetativo del frijol tiene un promedio de tres meses, por lo cual en un año se podrían obtener unas cuatro generaciones $\left(\mathrm{F}_{4}\right)$.

\section{Descripción de las variedades utilizadas en la primera parte del estudio}

Talamanca: Es una planta arbustiva y erecta de porte bajo, con una altura de 50 a $55 \mathrm{~cm}$, resistente al volcamiento y de maduración uniforme. Florece a los treinta y nueve días y la maduración ocurre a los setenta y dos días; su ciclo de vida es de ochenta días. Es un grano de color negro brillante y de producción alta (Monge, 1989).

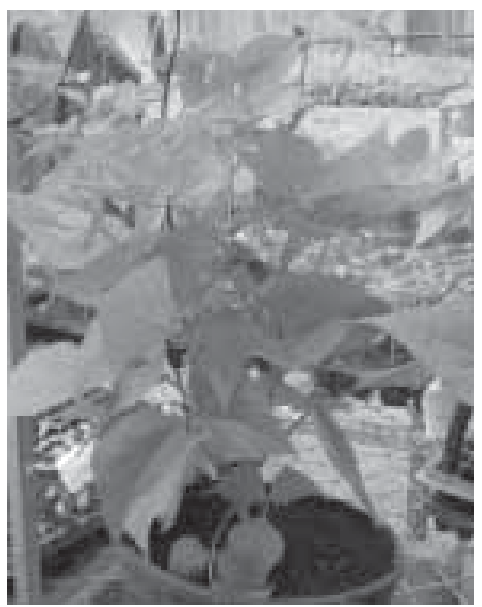

Imagen I.Variedad Talamanca. 
Brunca: Es una variedad con un hábito de crecimiento indeterminado y postrado, conocido anteriormente como de semiguía. Su altura es de $45 \mathrm{~cm}$. Una de sus principales características es su gran precocidad, ya que florece entre los treinta y cuatro y treinta $y$ siete días. Madura a los sesenta y seis días y su ciclo de cultivo es de setenta y cinco días. El ciclo es de 35 días a la floración y 72 a 75 días a la cosecha. Hábito de crecimiento tipo III postrado indeterminado (semiguía). Peso de 100 semillas: 20-22 g. Granos por vaina: 6. Es susceptible a Mustia, Mancha angular y Antracnosis; tolerante a la sequía. El grano es de color negro opaco.

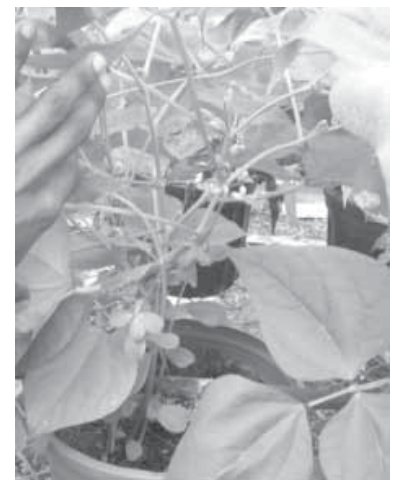

Imagen 2. Variedad Brunca.

Guaymí: El ciclo de esta variedad es de 38 días a la floración y de 74 a 76 días a la cosecha. Su hábito de crecimiento es del tipo llb semierecto indeterminado (semiguía). Peso de 100 semillas: 21-22 g. Granos por vaina: 6-7. Es susceptible a Antracnosis y de resistencia intermedia a la Mustia y Mancha Angular. El grano es de color negro brillante.

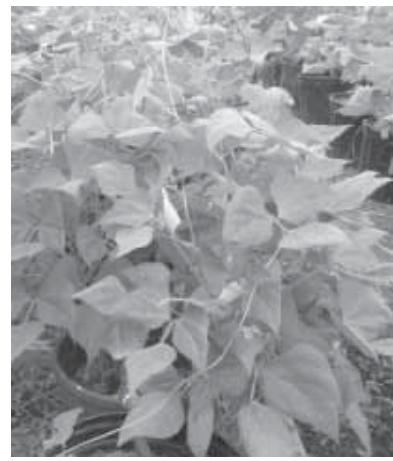

Imagen 3.Variedad Guaymí.
Negro Chapingo: El ciclo total de esta variedad es de 110 a 120 días; las primeras flores se presentan entre los 45 y 50 días, el periodo de floración a llenado de vainas tarda 45 a 55 días. El color de la flor es morado, la vaina es verde cuando aún no ha madurado y amarillo claro cuando está madura, la semilla es negra opaca; la planta tiene un hábito de crecimiento postrado, con follaje espeso y guías intermedias y largas que cierran los surcos; presenta gran carga productiva.

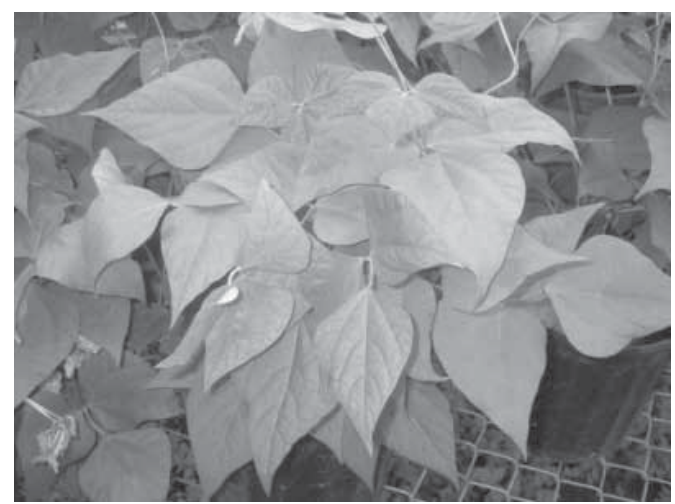

Imagen 4.Variedad Negro Chapingo.

\section{Materiales y métodos}

\section{Material experimental}

La obtención de una variedad mejorada (híbrida) apta para ser cosechada mediante máquinas cosechadoras se puede lograr mediante varios métodos de mejoramiento genético, tanto convencionales o clásicos como no convencionales o biotecnológicos, los cuales no se mencionarán en este trabajo.

Para lograr la variedad deseada se recurrió al mejoramiento clásico o convencional, que sigue los pasos de: "Introducción, hibridación y selección".

Se introdujeron variedades o líneas puras provenientes de la Universidad de Costa Rica (UCR) y de la Universidad Autónoma de Chapingo (UACH, México), que presentaban algunas de las condiciones posibles para ser utilizadas para la obtención de variedades adaptadas al área en estudio. Se evaluaron tres de las variedades "criollas" de alta producción adaptadas a la zona y explotadas por el productor. 
También se solicitó al Centro Internacional de Agricultura Tropical (CIAT) de Colombia material arbustivo (tipo I) y de floración determinada pero no se recibió ninguna respuesta de esta organización.

Para este fin se introdujo de la Universidad Autónoma de Chapingo la variedad semiarbustiva y de crecimiento indeterminado Tipo $\|$ b, denominada "Negro Chapingo" y se seleccionaron las variedades nacionales de color negro Guaymí y Brunca de crecimiento indeterminado Tipo II y III, estas dos últimas de la estación experimental Fabio Baudrit de la Universidad de Costa Rica, muy utilizadas por los productores de frijol de la zona norte y por el resto del país. Posteriormente, y debido a la ausencia de material arbustivo tipo I, se obtuvo de la UCR la variedad Talamanca, la cual presenta un crecimiento semi arbustivo. Esta variedad se combinó con las otras variedades como padre y también como madre en cruces recíprocos.

\section{Equipo utilizado}

Se utilizó un invernadero con techo de plástico y paredes de plástico antiáfidos, piso de piedra cuarta y mesas con marco de metal forrado con malla ciclón sobre bases de tubo galvanizado movibles y a un metro sobre el suelo. En dichas mesas se colocaron tarros de plástico de tamaño mediano, 10 tarros por variedad utilizada. Primero se inició el trabajo en un invernadero, el cual tuvo riego por goteo del 9 de julio al 24 de setiembre del 2007. A partir del 27 de setiembre se continuó el estudio en un segundo invernadero con las mismas condiciones anteriores, excepto el piso, que era de cemento, con riego al inicio por manguera y casi al final del proyecto con riego por aspersión.

Se incluyó también el equipo necesario para las polinizaciones dirigidas: pinzas de punta fina, pinceles finos, agujas grandes con mango de madera, tijeras pequeñas, lupas de mano, recipientes de plástico con cierre a presión, bandejas planas y hondas, un recipiente con alcohol gel y talco para niños, agregándose además hilos de colores diferentes, una bitácora de campo y bolsas de papel manila de diferentes tamaños.

\section{Manejo del material experimental}

Las plantas de frijol en los tarros, los cuales se llenaron con un sustrato a base de abono orgánico y arena, se fertilizaron cada vez que se reiniciaba un ciclo vegetativo; los ciclos fueron, iniciando con el primer ciclo, que se sembró el 9 de julio del 2007 y se terminó en el mes de setiembre del mismo año. El segundo ciclo se inició el 12 de octubre y culminó en diciembre del 2007, el tercer ciclo se inició el 13 de febrero del 2008 y culminó en junio de ese año.

Los ciclos de polinización fueron el 31 de agosto, el 5 de diciembre del 2007 y el 5 de marzo del 2008. Al inicio de cada ciclo de siembra se fertilizaron las plantas con 10- | $5 \mathrm{gr} /$ tarro de la fórmula |3-0-44; el control de malezas se realizó manualmente. Durante el experimento no se presentaron enfermedades graves, excepto por un ataque de ácaros, el cual fue controlado con la aplicación de Vertimec $®$ en dosis de I cc/litro de agua en dos ocasiones.

\section{Definición de etapas}

Se determinó que el proyecto se realizaría en dos etapas metodológicas, las cuales se describen a continuación:

\section{Primera etapa}

Introducción de las variedades que se utilizan en este proyecto y que no existen en el país. Las variedades introducidas se sembraron en potes de plástico/ variedad y se colocaron en mesas de metal dentro de un invernadero. Cada variedad participante en el cruce se cosechó manualmente en forma separada, identificándose las líneas obtenidas/cruce comúnmente llamadas híbridos FI.

Segunda etapa

Los "híbridos" resultantes de los cruces (FI) se identificaron de acuerdo con el Sistema Internacional, guardándose en sobres de papel debidamente identificados, para luego ser evaluados fenotípicamente (selección) a nivel de campo y verificar su comportamiento genético (segregación) en la segunda generación (F2), utilizando para ello los descriptores morfológicos descritos por el IPGRI (200I).

Los parentales donadores o aceptores del polen fueron seleccionados de las variedades productoras más usadas en la Zona Norte por los agricultores y cruzadas entre sí, utilizándose el método del cruce simple con las variedades introducidas.

\section{Procedimiento de polinización}

Se realizaron cruces dirigidos en dos vías: primero entre la variedad introducida (padre) sobre las variedades nacionales que serán las madres 
receptoras del polen, y luego se cruzaron también a la inversa, la variedad introducida como receptora (madre) y las variedades nacionales como donadoras de polen (padres).

Se emasculó (castró) la flor hembra y se procedió a la polinización artificial o dirigida, utilizando pinceles finos untados de polen de las variedades donadoras; luego estas flores fueron identificadas por hilos de colores, un color por cada variedad participante en el proceso.

Las flores fueron emasculadas (castradas) en preantesis temprana, utilizando para ello pinzas de punta fina para extirpar con facilidad las anteras de la flor receptora o madre. Las flores polinizadas no se cubrieron con bolsas debido a que se trata de plantas autógamas, las cuales son líneas puras y no se mezclan entre sí por ser de autopolinización.

La semilla híbrida se recolectó una vez que la vaina logró su maduración total o de campo, así como el amarillamiento total y el secamiento.

La recolección de polen se efectuó en la etapa fenológica de la antesis, recolectándose las anteras maduras y depositándose en recipientes plásticos. La polinización dirigida o artificial se realizó en horas de la mañana, para evitar la evapotranspiración potencial de las flores y así garantizar la posibilidad de un cruce exitoso. Se utilizó polen fresco y no se guardó para otro día con el fin de evitar la pérdida de fertilidad del mismo, además de que no se cuenta con equipo especial para tal efecto.

\section{Resultados y discusión}

Introducción de variedades de porte arbustivo

La introducción en el proyecto del material arbustivo (crecimiento tipo I) y de floración determinada fue imposible debido a la falta de contactos eficientes, pues desde la Universidad Autónoma de Chapingo en México se envió solo una variedad, la Negro Chapingo de grano negro que no presentó las características deseadas para la obtención de los genes necesarios para lograr obtener el tipo requerido.

Se le solicitó al CIAT de Colombia material de frijol con las características citadas y nunca se obtuvo respuesta ni se recibió material para ser utilizado en el proyecto.
Se recurrió a la Estación Experimental Fabio Baudrit de la Universidad de Costa Rica, que mantiene una copia de frijol del CIAT, pero no tenían el material genético adecuado para utilizarlo en el trabajo en cuestión; solo se recibió la variedad nacional Talamanca, la cual se considera semiarbustiva (tipo II) y de floración indeterminada, pero no reunió las características deseadas para la obtención de un híbrido arbustivo para cosecha mecanizada en la región.

También se entabló comunicación con el CATIE, sin embargo, esta institución no disponía del material necesario para realizar el cruzamiento. En resumen, el país no cuenta con este tipo de material, por lo tanto se debe introducir para futuros trabajos de mejoramiento genético.

\section{Hibridación dirigida o artificial mediante cruces recíprocos}

Durante el estudio se realizaron cuatro periodos de polinizaciones; el primero se inició el 10 de setiembre del 2007 y el último culminó el 23 de junio del 2008. Durante este trabajo se efectuaron 34 polinizaciones dirigidas entre los siguientes materiales:

\begin{tabular}{|c|c|c|}
\hline I. Brunca $\times$ Guaymí & $(\mathrm{B} \times \mathrm{G})$ & 5 cruces \\
\hline 2. Guaymí $\times$ Brunca & $(\mathrm{G} \times \mathrm{B})$ & 4 cruces \\
\hline 3. Guaymí $\times$ Talamanca & $(\mathrm{G} \times \mathrm{T})$ & 21 cruces \\
\hline 4. Talamanca $\times$ Guaymí & $(\mathrm{T} \times \mathrm{G})$ & 22 cruces \\
\hline 5. Brunca $\times$ Talamanca & $(\mathrm{B} \times \mathrm{T})$ & 58 cruces \\
\hline 6. Talamanca $\times$ Brunca & $(\mathrm{T} \times \mathrm{B})$ & 41 cruces \\
\hline 7. Talamanca $\times$ Chapingo & $(\mathrm{T} \times \mathrm{CH})$ & 44 cruces \\
\hline 8. Chapingo $\times$ Talamanca & $(\mathrm{Ch} \times \mathrm{T})$ & 52 cruces \\
\hline 9. Chapingo $\times$ Guaymí & $(\mathrm{Ch} \times \mathrm{G})$ & 15 cruces \\
\hline 10. Guaymí $\times$ Chapingo & $(\mathrm{G} \times \mathrm{Ch})$ & 14 cruces \\
\hline 11. Chapingo $\times$ Brunca & $(\mathrm{Ch} \times \mathrm{B})$ & 28 cruces \\
\hline 12. Brunca $\times$ Chapingo & $(\mathrm{B} \times \mathrm{Ch})$ & 37 cruces \\
\hline TOTAL & & 34 cruces \\
\hline
\end{tabular}

Uno de los principales problemas encontrados para el éxito del total de las polinizaciones dirigidas, que fue de solo el 50\%, fue la temperatura en el invernadero, la cual se mantuvo en promedio durante todo el periodo experimental en $34-35^{\circ} \mathrm{C}$ y eso afectó seriamente el resultado del experimento. Se registró 
Cuadro I. Polinizaciones realizadas entre las variedades participantes en los cruces para la obtención de un híbrido de porte arbustivo para cosecha mecánica. San Carlos 2008.

\begin{tabular}{|c|c|c|c|c|}
\hline$N^{\circ}$ Polinización & Cruces & Total & Cuaje & Híbrido obtenido FI \\
\hline \multirow{4}{*}{ I } & $\mathrm{Ch} \times \mathrm{B}$ & 10 & & \\
\hline & $\mathrm{B} \times \mathrm{Ch}$ & 14 & 1 & $\mathrm{BCh}$ \\
\hline & $\mathrm{G} \times \mathrm{Ch}$ & 8 & & \\
\hline & $\mathrm{Ch} \times \mathrm{G}$ & 8 & & \\
\hline \multirow{3}{*}{ ॥ } & $G \times T$ & 2 & & \\
\hline & $\mathrm{Ch} \times \mathrm{T}$ & 2 & & \\
\hline & $\mathrm{B} \times \mathrm{T}$ & II & & \\
\hline \multirow{14}{*}{ III } & $G \times B$ & 3 & & \\
\hline & $B \times G$ & 5 & & \\
\hline & $G \times T$ & 6 & & \\
\hline & $T \times G$ & 4 & & \\
\hline & $\mathrm{T} \times \mathrm{B}$ & 11 & & \\
\hline & $\mathrm{B} \times \mathrm{T}$ & 23 & I & ChT \\
\hline & $\mathrm{Ch} \times \mathrm{T}$ & 27 & 1 & TCh \\
\hline & $\mathrm{T} \times \mathrm{Ch}$ & 21 & & \\
\hline & $\mathrm{Ch} \times \mathrm{G}$ & 7 & & \\
\hline & $\mathrm{G} \times \mathrm{Ch}$ & 6 & & \\
\hline & $\mathrm{Ch} \times \mathrm{B}$ & 14 & & \\
\hline & $\mathrm{B} \times \mathrm{Ch}$ & 19 & & \\
\hline & $G \times T$ & 13 & & \\
\hline & $T \times G$ & 18 & & \\
\hline \multirow{7}{*}{ IV } & $\mathrm{B} \times \mathrm{T}$ & 24 & 1 & BT \\
\hline & $T \times B$ & 30 & 1 & $\mathrm{~TB}$ \\
\hline & $G \times B$ & 1 & & \\
\hline & $\mathrm{Ch} \times \mathrm{B}$ & 4 & & \\
\hline & $\mathrm{B} \times \mathrm{Ch}$ & 4 & & \\
\hline & $\mathrm{Ch} \times \mathrm{T}$ & 23 & & \\
\hline & $\mathrm{T} \times \mathrm{Ch}$ & 23 & & \\
\hline Total & & 341 & 5 & \\
\hline
\end{tabular}

cerca de un 30\% de aborto de las vainas (102 cruces), producto de los cruces realizados y un $20 \%$ en la obtención de vainas vanas (68 cruces) como resultado del efecto negativo de la temperatura en el cuaje de los cruces.

El proceso de polinización es muy susceptible a los cambios climatológicos imperantes en el campo, pues una temperatura superior al promedio (28$30^{\circ} \mathrm{C}$ ) impide el crecimiento del tubo polínico y la fecundación de la célula huevo, ya que hay desecación del pistilo y se marchitan las estructuras sexuales; esta es una de las principales implicaciones. Otra causa del "vaneo", presencia de vainas vacías, es la pérdida de agua en los tejidos del ovario y déficit hídrico para las síntesis de carbohidratos en la formación del embrión (Flores, 1994).

Estos cálculos se realizaron relacionando los conteos de flores polinizadas naturalmente por autopolinización con los resultados de las polinizaciones artificiales 0 dirigidas realizadas durante el estudio.

Como solución al problema de la alta temperatura y la baja humedad relativa dentro del invernadero, se procedió a cambiar el riego con manguera por el de aspersión aérea, el cual se instaló muy cerca del final del proyecto.

La identificación de cada una de las polinizaciones se realizó de acuerdo con las disposiciones internacionales. El primer nombre es la variedad aceptora o receptora de polen, o sea la madre, y el segundo nombre es el del donador de polen, es decir, el padre $\left(+\times \delta^{\Uparrow}\right)$.

En el siguiente cuadro se muestran los diferentes cruces realizados y los híbridos $\mathrm{Fl}$ obtenidos durante todo el periodo experimental del proyecto de investigación. 


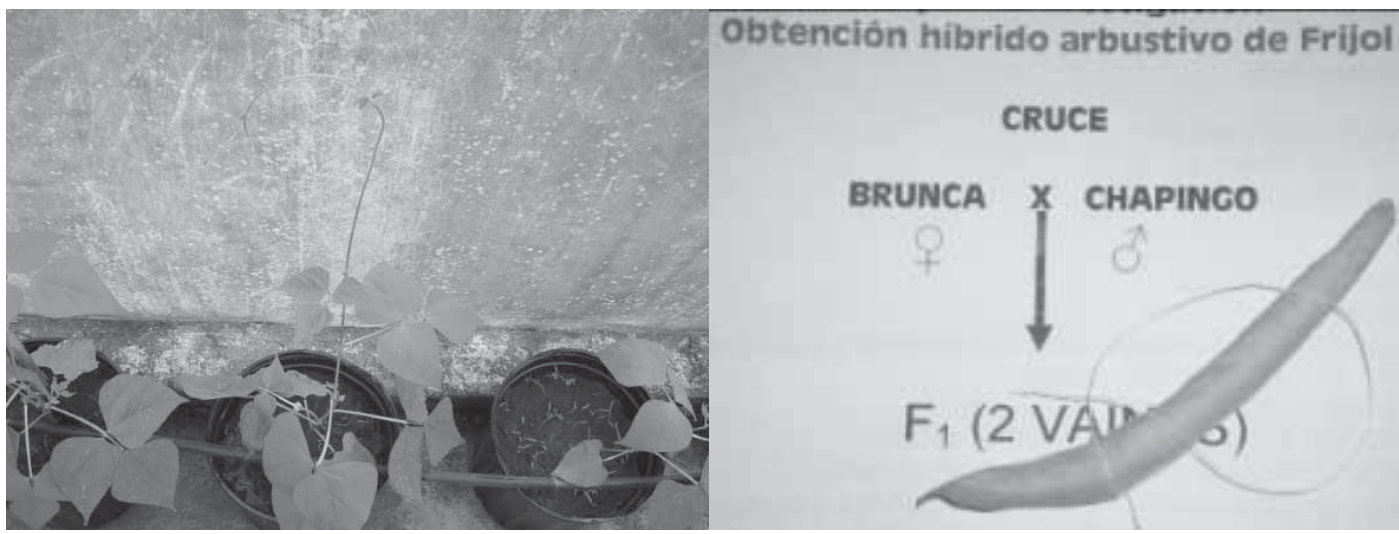

Imagen 5. Híbrido Fl: (Bch), cruce entre Brunca × Chapingo. Proyecto de Investigación TEC, San Carlos 2008.

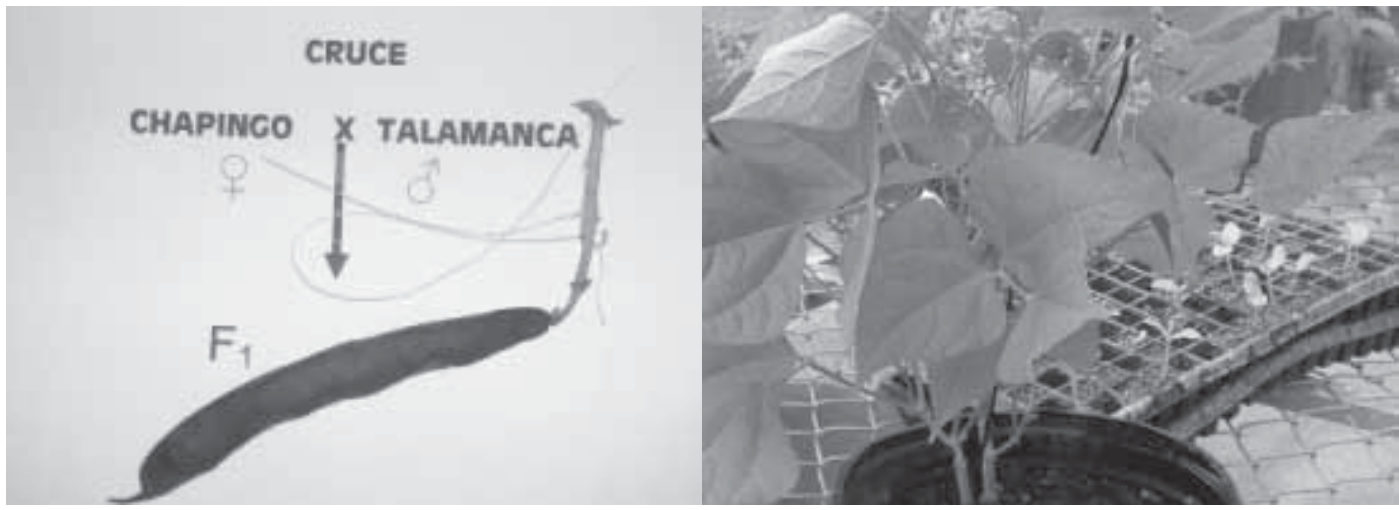

Imagen 6. Híbrido FI: (ChT), cruce entre Chapingo x Talamanca. Proyecto de Investigación TEC, San Carlos, 2008.

Selección individual y evaluación del material híbrido segregante en la F2

Se evaluaron los híbridos obtenidos solamente durante una sola generación, debido a la finalización del presupuesto y del proyecto de investigación. Generalmente, los ciclos vegetativos de las variedades evaluadas en este estudio fueron de entre 75 y 120 días (2,5-4 meses).

El primer híbrido obtenido del cruce entre la variedad $\mathrm{Fl}$ : Brunca $\times$ Chapingo (BCh) se sembró para su evaluación a nivel de invernadero el 20 de febrero del 2008. Se sembraron cuatro semillas, dos por macetera, quedando material para otras pruebas adicionales. La floración se inició el 13 de marzo del 2008 y su cosechado se hizo el día 30 de abril del mismo año, obteniéndose la segunda generación o F2. No se detectaron líneas segregantes recesivas con características diferentes a la de sus padres en la primera población de la F2.
Las características del híbrido F2 fueron las típicas de un material no arbustivo y de floración indeterminada y con líneas guías largas, no apto para la cosecha mecanizada (imagen 5).

No se pudo realizar la siembra de la segunda generación (F2) para lograr la F3 y las sucesivas generaciones, debido a la finalización técnica del proyecto.

El segundo híbrido $\mathrm{FI}$ que se obtuvo fue el cruce entre las variedades Chapingo x Talamanca (ChT). Este segundo híbrido no fue sembrado por razones de tiempo pero partiendo del resultado de la prueba del primer híbrido evaluado (BCh), se puede decir que la segregación en la F2 sería igual que la anterior por tratarse de líneas puras de autopolinización, presentando características no deseadas para utilizarse como material para obtener una variedad arbustiva para la cosecha mecanizada, pues su porte será semiarbustivo con líneas guías cortas pero 
de floración indeterminada, igual que los padres participantes en el cruce (imagen 6).

Los híbridos FI siguientes obtenidos producto de la polinización dirigida: $\mathrm{TCH}, \mathrm{BT}$ y $\mathrm{TB}$, no se pudieron evaluar a nivel de invernadero por la culminación del proyecto. Los granos de los anteriores híbridos se encuentran en la Escuela de Agronomía del TEC, sede regional, para ser evaluados en cualquier momento.

Otra de las razones por las cuales no se evaluaron fue que, igual que los anteriores híbridos ChT y $\mathrm{BCh}$, en las siguientes generaciones presentarán las mismas características no compatibles para una variedad arbustiva (tipo I) y de floración determinada, características necesarias y buscadas para la obtención de una variedad apta para ser cosechada en forma mecánica y objetivo primordial de este estudio.

Las plantas autógamas (autopolinización) se consideran líneas puras, eso significa que existe una gran homogeneización de los caracteres genéticos a lo largo del tiempo, los cuales no tienden a segregar en las futuras generaciones. El caso contrario son las plantas de polinización libre o cruzada, llamadas alógamas, las cuales son heterocigotos y en cada generación hay segregación, debido a la heterosis o vigor híbrido presente en ellas (Muñoz, 2008).

Al realizar cruces entre las plantas autógamas, se está trabajando con material homocigoto. El ingreso de genes del mismo material existente en las variedades participantes va a predominar en sus descendencias en la segunda generación o F2. Posiblemente en generaciones siguientes (F3, F4, F5...), por las recombinaciones al azar, podrían aparecer caracteres diferentes provenientes de las variedades involucradas, aunque, como se describió anteriormente, todo el material genético participante en el proyecto es igual, no hay características de plantas arbustivas y de floración determinada, necesarias para la obtención de plantas arbustivas para la cosecha mecanizada; esta es una razón importante para evaluar solo uno de los híbridos logrados en este trabajo de investigación; además, no existió la posibilidad de seguir evaluando el material obtenido por la conclusión técnica del proyecto.

La llegada reciente de material genético proveniente del Ministerio de Agricultura de República
Dominicana, enviado por el Ingeniero Julio C. Nin, abre la posibilidad de continuar trabajando en esa dirección hasta obtener la variedad deseada, trabajo que se realizará mediante tesis de estudiantes. El ingeniero y genetista Julio Nin, basado en el marco del Convenio de la FAO, me ha enviado dos variedades de porte arbustivo Tipo I y floración determinada: variedades "Idiaf Yaconin (conocida como "Giacomela grano corto") y la variedad PC50, (conocida como "Checa"). Con dicho material y las variedades nacionales Brunca y Guaymí, se continuará la investigación hasta lograr obtener las variedades arbustivas para cosecha mecánica para la zona norte de Costa Rica (segunda parte).

\section{Conclusiones}

I. Se pudo dominar la técnica de la polinización dirigida o artificial con éxito al realizarse más de 300 cruces.

2. Hubo éxito al lograrse cinco híbridos en la primera generación (FI), estos híbridos están en capacidad de ser sembrados en el campo para una valoración posterior y un análisis fenotípico más exhaustivo mediante la selección en la segunda generación o F2 y sucesivas.

3. Se entrenó a estudiantes en la técnica y se espera continuar con ellos el trabajo mediante las prácticas de especialidad y tesis de grado en la Escuela de Agronomía.

\section{Agradecimiento}

Un agradecimiento muy especial a los estudiantes que participaron en el proyecto: Allan Zúñiga Morales, estudiante de la escuela de Agronomía del Tecnológico de Costa Rica, y a las estudiantes cooperantes de la Universidad de Chapingo en México: Fernanda Citlalli Calderón Soto y Georgina Ithandehui Ávila Castañeda. Dichos estudiantes participaron activamente en las polinizaciones y en otras actividades relacionadas con el proyecto durante un periodo de tres meses del 2007. Su apoyo fue muy valioso para el proyecto de investigación. 


\section{Bibliografía}

Flores, V.E. (1994). La Planta: Estructura y Función. Cartago: Editorial Tecnológica de Costa Rica. 2 edición. 501 p.

IPGRI-CGIAR. 200I. Descriptores morfológicos para Phaseolus vulgaris. http://www.ipgri.cgiar.org

Jiménez, M.C. (1990). Aspectos Agroecológicos y Zonificación del cultivo de frijol (Phaseolus vulgaris). San José: Secretaria Ejecutiva de Planificación Sectorial Agropecuaria (SEPSA). 55 p.

Ledesma, A.E. (2000). Situación actual de los cultivos de frijol y arroz. Consejo Nacional de la Producción Región Huetar Norte. 40 p.

López, M.; Fernández, F. \& Schoonhoven, A. (1985). Frijol: Investigación y Producción. Colombia: PNUD, CIAT. 417 p.
Mateo, B.J.M. (196I). Leguminosas de grano. La Habana: Editorial Revolucionaria, Instituto del Libro. 550 p.

Monge, J.C. ( I 989). El cultivo de frijol. San José: Edit. EUNED. $192 \mathrm{p}$.

Muñoz, R.C. (2008). Mejoramiento genético de los cultivos. Cartago: Editorial Tecnológica de Costa Rica. 166 p.

Poehlman, J.M. (1987). Mejoramiento genético de las cosechas. México: Edit. Limusa. 453 p.

Rojas, J.C. (1998). El cultivo del frijol común en América tropical. Honduras: El Zamorano. 52 p.

Salazar, R.J. (2004). La actividad del frijol en Costa Rica durante el año 2004. VIII Reunión Anual del Sector Frijolero de Costa Rica. PITTA-Frijol. 
Tecnología en Marcha,

32 Vol. 25, N², Abril-Junio 2012 